\title{
On Multiple Prime Divisors of Cyclotomic Polynomials
}

\author{
By Wayne L. McDaniel
}

\begin{abstract}
Let $q$ be a prime $<150$ and $F_{n}$ be the cyclotomic polynomial of order $n$. All triples $(p, n, q)$ with $p$ an odd prime $<10^{6}$ when $q<100$ and $p<10^{4}$ when $100<q$ $<150$ are given for which $F_{n}(q)$ is divisible by $p^{t}(t>1)$.
\end{abstract}

1. Introduction. The cyclotomic polynomial $F_{n}$ of order $n$ is defined by

$$
F_{n}(x)=\prod_{k}\left(x-e^{2 \pi i k / n}\right)
$$

where the index $k$ ranges over the integers relatively prime to $n$. A basic formula relating $x^{n}-1$ to the cyclotomic polynomials [3, Chapter 8$]$ is

$$
x^{n}-1=\prod_{d \mid n} F_{d}(x) .
$$

Certain investigations, such as, for example, those concerned with odd perfect numbers and amicable numbers draw upon a knowledge of the prime divisors of $F_{n}(q)$, for $q$ prime; frequently, a knowledge of whether $F_{n}(q)$ is free of relatively small factors of multiplicity greater than one is helpful. We present in this paper all triples $(p, n, q)$ with $p$ an odd prime less than $L$ ( $L$ defined below), $q$ a prime less than 150 and $n$ any positive integer, for which a power of $p$ greater than the first divides $F_{n}(q)$.

We have made extensive use of the tables of solutions of $a^{p-1} \equiv 1\left(\bmod p^{2}\right)$ presented in papers by Brillhart, Tonascia and Weinberger [1], and Riesel [4]. Our search limits for $p$ are those given in these papers; if $q$ is a prime $<150$, then $p<L$ for $L$ defined as follows:

$$
\begin{array}{rlrl}
q & =2 & & L=3 \cdot 10^{9} \\
q & =3 & & L=2^{30} \\
q & =5 & L & =2^{29} \\
q & =7,11,13,29,49 & & =2^{28} \\
q & =17,19 & L & =2^{27} \\
q & =23 & L & =2^{26} \\
q & =61,73,89,97 & & L=2^{25} \\
q & =31,37,41,43,53,59,67,71,79,83 & & L=10^{6} \\
100 & <q<150 & & L=10^{4}
\end{array}
$$

Received August 27, 1973.

AMS (MOS) subject classifications (1970). Primary 10A25; Secondary 10A40.

Key words and phrases. Cyclotomic polynomial, sum of divisors.

Copyright $\odot$ 1974, American Mathematical Society 
2. The Approach. That starting with the available solutions of the congruence $a^{p-1} \equiv 1\left(\bmod p^{2}\right)$ leads to a most efficient means of finding the multiple odd prime factors of $F_{n}(a)$, for any positive integer $n$, is based on the following reasoning: It is well known (see [2, pp. 164, 166]) that $F_{n}(a)$ has as possible divisors the largest prime factor of $n$ (but not its square if $n>2$ ) and numbers of the form $1+k n$. If, now, $p^{t}(t>1)$ is an odd prime power divisor of $F_{n}(a), n$ any positive integer, then $p-1=k n$ for some integer $k$; since, by $(2), F_{n}(a)$ divides $a^{n}-1$, it is clear that $p^{t}$ divides $a^{n}-1$, and, therefore, $a^{p-1} \equiv 1\left(\bmod p^{t}\right)$. It follows that the only possible odd prime power divisors $p^{t}(t>1)$ of $F_{n}(a)$, for $p<L$ and $a<150$, are those primes $p$ listed in the tables of [1] and [4].

We have restricted our investigation to $F_{n}(a)$ for $a$ a prime largely because interest in the multiplicity of divisors of cyclotomic polynomials frequently occurs in connection with their appearance as factors of the sum-of-divisors function $\sigma$. Since $\sigma$ is a multiplicative function and, for $q$ prime,

$$
\sigma\left(q^{n-1}\right)=\left(q^{n}-1\right) /(q-1)=\prod_{d \mid n} F_{d}(q), \quad d \neq 1,
$$

it is sufficient to confine one's attention to $F_{n}(a)$ for $a$ a prime.

Our calculation, carried out on the University of Missouri's IBM 360, was shortened through application of the following extension of Theorem 4 in [1]:

THEOREM. Let $a, r$ and $m$ be positive integers with $(m, \varphi(m))=1$. If a belongs to $e(\bmod m)$ and $a^{\varphi(m)} \equiv 1\left(\bmod m^{r}\right)$, then a belongs to $e\left(\bmod m^{r}\right)$.

Proof. The proof is by mathematical induction on $r$. The theorem is trivially true when $r=1$. If the theorem is assumed to be true for $r=t$, then $a^{e}=1+\mathrm{km}^{t}$ for some positive integer $k$. Now, when $r=t+1$,

$$
\begin{aligned}
1 & \equiv a^{\varphi(m)} \equiv\left(a^{e}\right)^{\varphi(m) / e}=\left(1+k m^{t}\right)^{\varphi(m) / e} \\
& \equiv 1+k m^{t} \varphi(m) / e\left(\bmod m^{t+1}\right),
\end{aligned}
$$

from which it follows that $m \mid k$. Hence, $a^{e} \equiv 1\left(\bmod m^{t+1}\right)$. No smaller power of $a$ is congruent to $1\left(\bmod m^{t+1}\right)$, since $a$ belongs to $e(\bmod m)$.

We immediately have this

COROLlaRY. If, for some odd prime $p$ and positive integers $a$ and $r$, a belongs to the exponent $e(\bmod p)$ and $a^{p-1} \equiv 1\left(\bmod p^{r}\right)$, then $p^{r} \mid F_{e}(a)$.

Proof. Since, by the Theorem, $p^{r}$ divides $a^{e}-1, p \mid F_{d}(a)$ for some divisor $d$ of $e$, by (2). But then, $p \mid a^{d}-1$, so $d=e$. Since $d=e$ is the only divisor of $e$ for which $p \mid F_{d}(a), p^{r}$ divides $F_{e}(a)$.

The obvious implication of the Corollary, with respect to the problem of finding $p, n$ and $q(p<L, q<150)$ such that $p^{t} \mid F_{n}(q)$, is that, for each pair $p$ and $q$ such that $q^{p-1} \equiv 1\left(\bmod p^{t}\right)(t=2$ or 3$)$ in the tables of [1] and [4], one need only find the smallest factor $n$ of $p-1$ for which $p \mid q^{n}-1$. It follows that $p^{t}$ divides $F_{n}(q)$.

Our procedure, then, was straightforward; the exponent to which $q$ belongs $(\bmod p)$ was found in the usual way. Only four values of $F_{n}(q)$ are divisible by $p^{3}$ for $p<L, q<150$, and these are marked with an asterisk in the table. No $F_{n}(q)$ is divisible by the fourth power of an odd prime for $p$ and $q$ in our ranges.

We are indebted to the referee for pointing out that the entry $a=23$, $p=1370377$ in Table I of [1] should have been $a=23, p=13703077$. Subsequently, we checked all values of $a$ and $p$ listed in the tables of both [1] and [4], and the 
triples in our own table, and found all entries to be correct with the one exception noted above.

All primes $p$ and $q, 2<p<L, q<150$, and integers $n$ for which $p^{2} \mid F_{n}(q)$.

\begin{tabular}{|c|c|c|c|c|c|}
\hline$p$ & $n$ & $q$ & $p$ & $n$ & $q$ \\
\hline 1093 & $2^{2} \cdot 7 \cdot 13$ & 2 & $* 3$ & 2 & 53 \\
\hline 3511 & $3^{3} \cdot 5 \cdot 13$ & 2 & 47 & 23 & 53 \\
\hline 11 & 5 & 3 & 59 & 29 & 53 \\
\hline 1006003 & $2 \cdot 3^{2} \cdot 55889$ & 3 & 97 & $2^{4} \cdot 3$ & 53 \\
\hline 20771 & $5 \cdot 31 \cdot 67$ & 5 & 2777 & $2^{2} \cdot 347$ & 59 \\
\hline 40487 & $2 \cdot 31 \cdot 653$ & 5 & 7 & 3 & 67 \\
\hline 53471161 & $2 \cdot 3^{2} \cdot 5 \cdot 148531$ & 5 & 47 & $2 \cdot 23$ & 67 \\
\hline 5 & $2^{2}$ & 7 & 268573 & $2 \cdot 3 \cdot 22381$ & 67 \\
\hline 491531 & $5 \cdot 13 \cdot 19 \cdot 199$ & 7 & 3 & 2 & 71 \\
\hline 71 & $2 \cdot 5 \cdot 7$ & 11 & 47 & 23 & 71 \\
\hline 863 & $2 \cdot 431$ & 13 & 331 & $3 \cdot 5 \cdot 11$ & 71 \\
\hline 1747591 & $3 \cdot 5 \cdot 13 \cdot 4481$ & 13 & 3 & 1 & 73 \\
\hline 3 & 2 & 17 & 7 & 3 & 79 \\
\hline 46021 & $2 \cdot 5 \cdot 13 \cdot 59$ & 17 & 263 & $2 \cdot 131$ & 79 \\
\hline 48947 & 24473 & 17 & 3037 & $2^{2} \cdot 3 \cdot 11 \cdot 23$ & 79 \\
\hline 3 & 1 & 19 & 4871 & 487 & 83 \\
\hline$* 7$ & $2 \cdot 3$ & 19 & 13691 & $5 \cdot 37^{2}$ & 83 \\
\hline 13 & $2^{2} \cdot 3$ & 19 & 3 & 2 & 89 \\
\hline 43 & $2 \cdot 3 \cdot 7$ & 19 & 13 & $2^{2} \cdot 3$ & 89 \\
\hline 137 & $2^{2} \cdot 17$ & 19 & 7 & 2 & 97 \\
\hline 63061489 & $2^{4} \cdot 3^{2} \cdot 7 \cdot 73 \cdot 857$ & 19 & 5 & 1 & 101 \\
\hline 13 & $2 \cdot 3$ & 23 & $* 3$ & 2 & 107 \\
\hline 2481757 & $2^{2} \cdot 206813$ & 23 & 5 & $2^{2}$ & 107 \\
\hline 13703077 & $2^{2} \cdot 3^{2} \cdot 380641$ & 23 & 97 & $2^{5} \cdot 3$ & 107 \\
\hline 7 & $2 \cdot 3$ & 31 & $* 3$ & 1 & 109 \\
\hline 79 & $3 \cdot 13$ & 31 & 3 & 1 & 127 \\
\hline 6451 & $3 \cdot 5^{2} \cdot 43$ & 31 & 19 & $2 \cdot 3^{2}$ & 127 \\
\hline 3 & 1 & 37 & 907 & $2 \cdot 3 \cdot 151$ & 127 \\
\hline 77867 & $2 \cdot 38933$ & 37 & 17 & $2^{4}$ & 131 \\
\hline 29 & $2^{2}$ & 41 & 29 & $2^{2} \cdot 7$ & 137 \\
\hline 1025273 & $2^{3} \cdot 128159$ & 41 & 59 & 29 & 137 \\
\hline 5 & $2^{2}$ & 43 & 6733 & $2^{2} \cdot 3 \cdot 11 \cdot 17$ & 137 \\
\hline 103 & $2 \cdot 3 \cdot 17$ & 43 & 5 & 2 & 149 \\
\hline
\end{tabular}


We remark that one can readily infer that if $q$ is a prime $<150$ and $n$ is any positive integer $>1$, then $\sigma\left(q^{n-1}\right)$ is square-free of prime divisors $p<L, p \nmid n$, except in those cases where $p, n$ and $q$ are listed in our table. This is a consequence of (3) and a theorem due to Sylvester [5] which states, essentially, that if $F_{r}(a)$ and $F_{s}(a)$ are distinct divisors of $\left(a^{n}-1\right) /(a-1)$, then, except for divisors of $r$ and $s$, $F_{r}(a)$ and $F_{s}(a)$ are relatively prime.

Department of Mathematics

University of Missouri-St. Louis

St. Louis, Missouri 63121

1. J. Brillhart, J. Tonascia \& P. Weinberger, "On the Fermat quotient," Proceedings of the 1969 Atlas Symposium on Computers in Number Theory (Oxford, 1969), pp. 213-222.

2. T. NAGELL, Introduction to Number Theory, Wiley, New York, 1951. MR 13, 207.

3. H. RADEMACHER, Lectures on Elementary Number Theory, Blaisdell, Waltham, Mass., 1964. MR 30 \#1079.

4. H. RIESEL, "Note on the congruence $a^{p-1} \equiv 1\left(\bmod p^{2}\right)$," Math. Comp., v. 18, 1964, pp. 149-150. MR 28 \# 1156.

5. J. J. Sylvester, "On the divisors of the sum of a geometrical series whose first term is unity and common ratio any positive or negative number," Nature, v. 37, 1888, pp. 417-418; Collected Mathematical Papers, v. 4, 1912, pp. 625-629. 